Supplementary information for:

\title{
Polymer-mediated adhesion: nanoscale surface morphology and failure mechanisms
}

\author{
Alberto Baggioli, Mosè Casalegno, Alessio David, Marta Pasquini and Guido Raos \\ Politecnico di Milano, Milano, Italy
}

Conversion from Lennard-Jones to conventional SI units.

The mapping from $\mathrm{U}$ to $\mathrm{SI}$ units requires a decision about the values attached to specific quantities, such as the bead diameter $\sigma$. The Kremer-Grest bead-and-spring model [1] is highly simplified. It was developed with computational simplicity in mind, without attempting to reproduce the properties of any specific polymer. The simplification is possible thanks to the universal behavior of polymers, at large length and long time scales. Its adaptation to specific polymers is possible, but it requires at least the introduction of some stiffness, through a bond bending energy.[2]

In view of the previous considerations, any mapping of the Kremer-Grest model will always be somewhat approximate. Nonetheless, adopting Kröger's suggested mapping for polystyrene,[3] we have the following conversion factors:

- Distance: $\sigma=0.97 \mathrm{~nm}$

- Mass: $\quad m=364 \mathrm{~g} / \mathrm{mol}$

- Energy: $\quad \varepsilon_{P P} / k_{B}=490 \mathrm{~K}$, or equivalently $\varepsilon_{P P}=4.07 \mathrm{~kJ} / \mathrm{mol}$.

- Density: $\quad m / \sigma^{3}=6.6 \mathrm{~g} / \mathrm{cm}^{3}=6.6 \mathrm{Mg} / \mathrm{m}^{3}$.

- Stress: $\quad \varepsilon p P / \sigma^{3}=7.4 \mathrm{MPa}$

- Time: $\tau=\sqrt{m \sigma^{2} / \varepsilon_{P P}}=9.1 \mathrm{ps}$

- Velocity: $\sigma / \tau=107 \mathrm{~m} / \mathrm{s}$

- Strain rate: $1 / \tau=0.11 \mathrm{ps}^{-1}$.

This mapping is approximate, also because in the present simulations we have adopted a slightly shorter FENE bond length [4] and we have included the attractive tail of the $\mathrm{L}$ interaction, which is absent in the conventional Kremer-Grest model. Nonetheless, the mapped parameters appear to be reasonable. The molecular mass of the polymer chains is $47 \mathrm{kDa}$. The initial inter-surface distance would be equal to $12.6 \mathrm{~nm}$. The "fast" and "slow" pulling speeds are equal to 10.7 and $1.17 \mathrm{~m} / \mathrm{s}$, respectively. The stresses recorded in the simulations are in the 1-10 MPa range.

[1] Kremer, K.; Grest, G. S. Dynamics of Entangled Linear Polymer Melts: A Molecular-dynamics Simulation. J. Chem. Phys. 1990, 92 (8), 5057-5086. https://doi.org/10.1063/1.458541

[2] Everaers, R.; Karimi-Varzaneh, H. A.; Fleck, F.; Hojdis, N.; Svaneborg, C. Kremer-Grest Models for Commodity Polymer Melts: Linking Theory, Experiment, and Simulation at the Kuhn Scale. Macromolecules 2020, 53 (6), 1901-1916. https://doi.org/10.1021/acs.macromol.9b02428

[3] Kröger, M. Simple Models for Complex Nonequilibrium Fluids. Phys. Rep. 2004, 390 (6), 453-551. https://doi.org/10.1016/j.physrep.2003.10.014

[4] MacKura, M. E.; Simmons, D. S. Enhancing Heterogenous Crystallization Resistance in a BeadSpring Polymer Model by Modifying Bond Length. J. Polym. Sci. Part B Polym. Phys. 2014, 52 (2), 134140. https://doi.org/10.1002/polb.23398 
Additional plots and figures
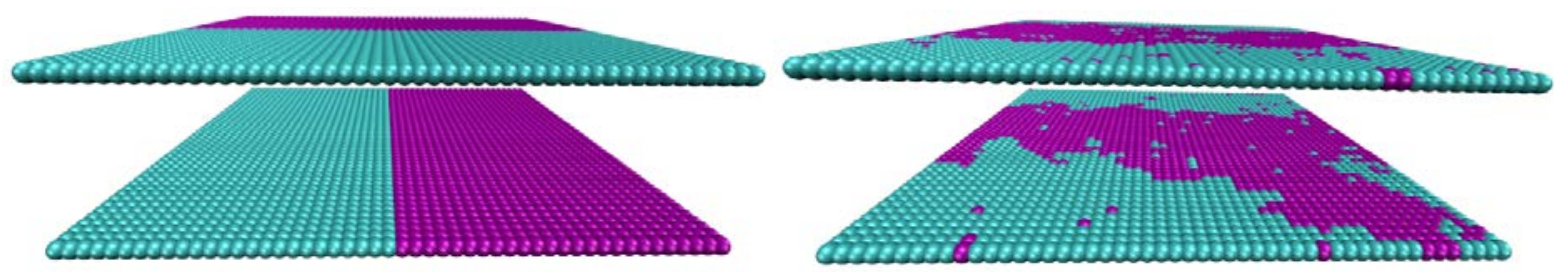

$\alpha=0.96$

$\alpha=0.79$

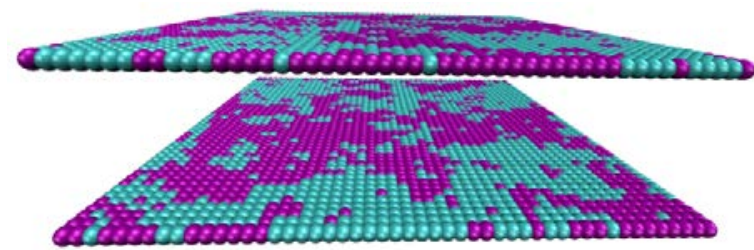

$\alpha=0.53$

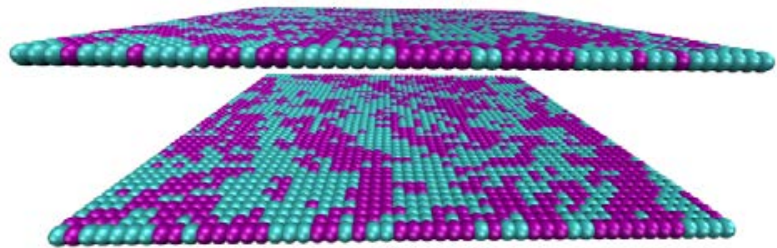

$\alpha=0.35$

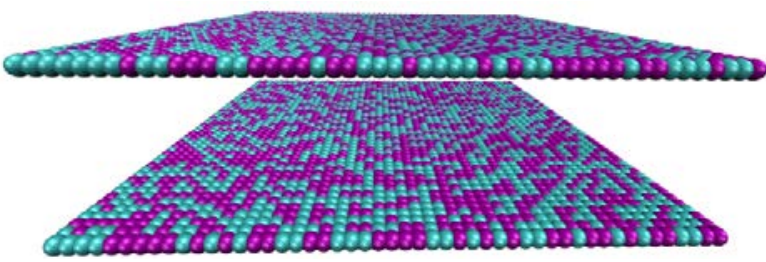

$\alpha=0.00$

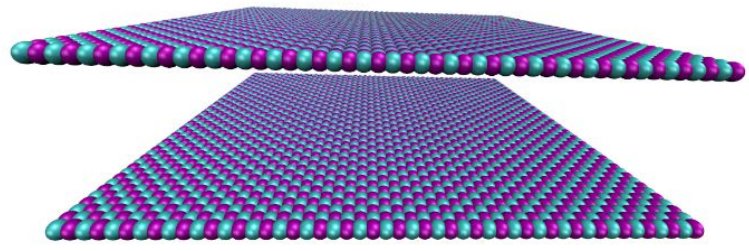

$\alpha=-1.00$

Figure SI.1: morphologies of the simulated surfaces.

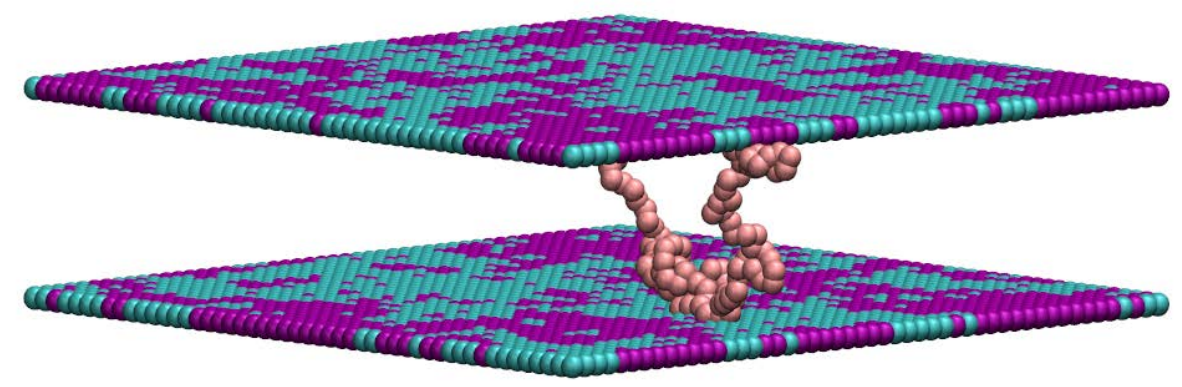

Figure SI.2: one bridging chain between two surfaces. 


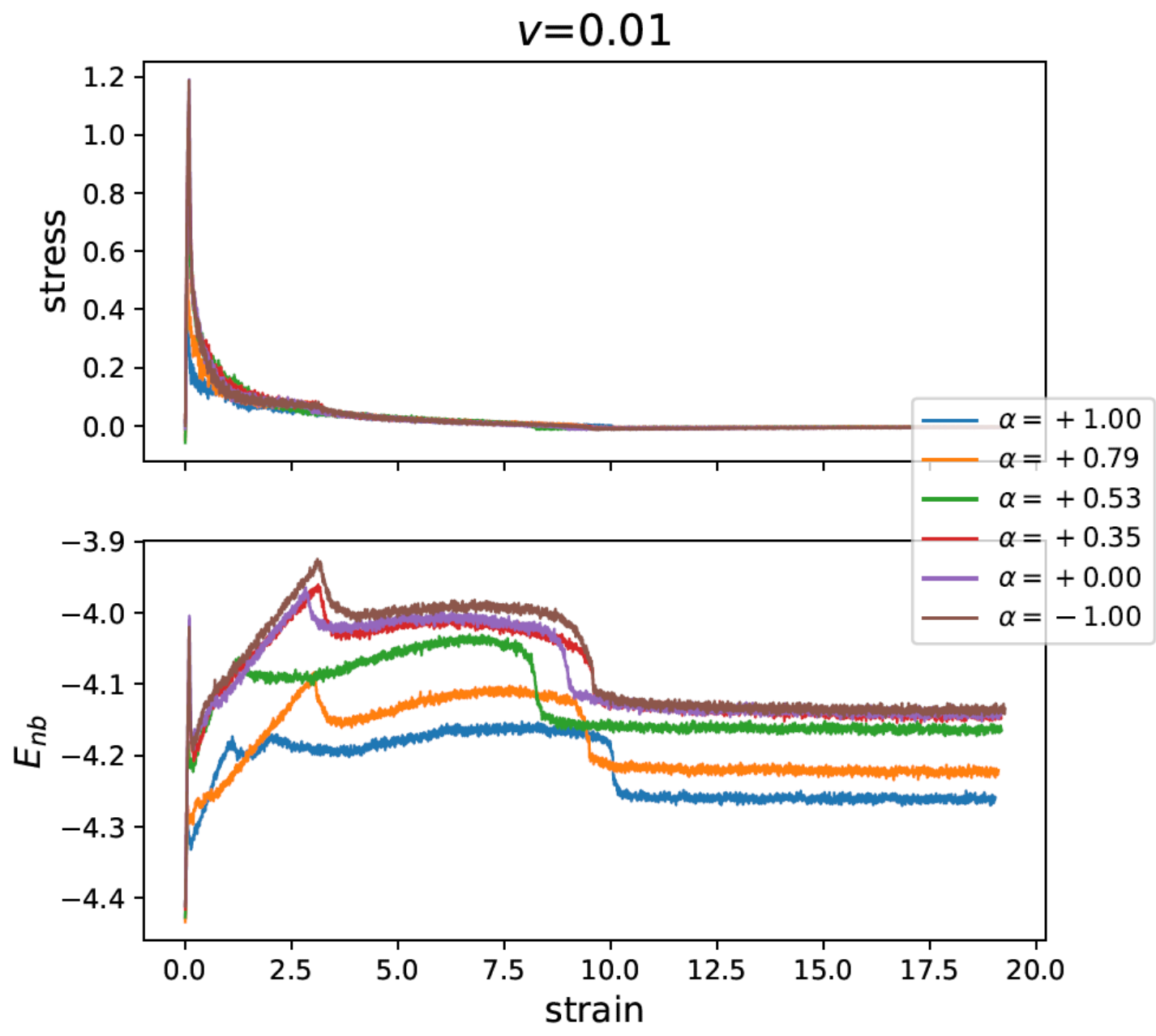

Figure SI.3: Full stress-strain curves and non-bonding energy plots for all systems, for "slow" pulling velocity $\left(\mathrm{v}=0.01\right.$, corresponding to $\left.\dot{\varepsilon}=7.7 \times 10^{-4}\right)$. 


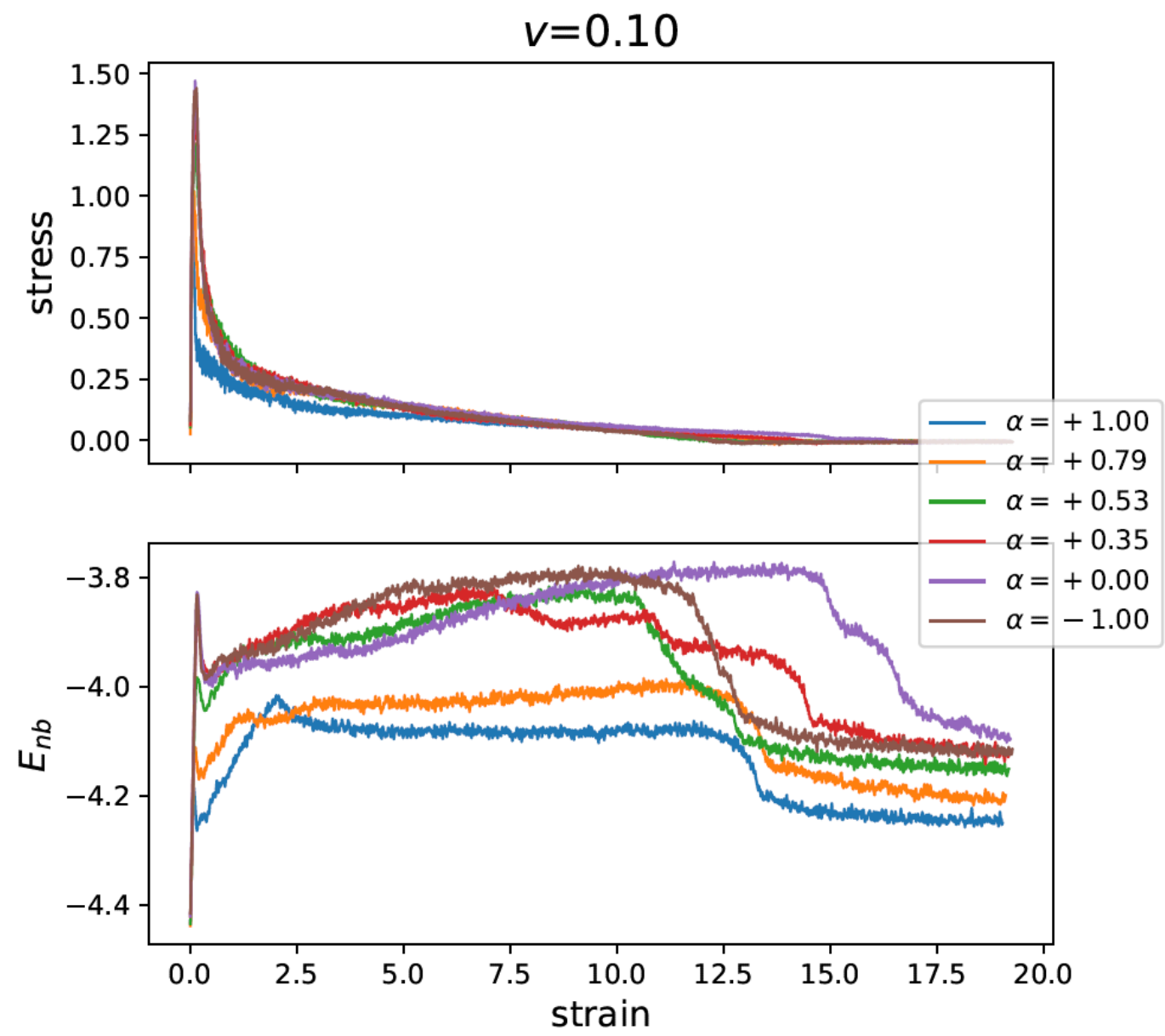

Figure SI.4: Full stress-strain curves and non-bonding energy plots for all systems, for "fast" pulling velocity ( $v=0.10$, corresponding to $\dot{\varepsilon}=7.7 \times 10^{-3}$ ). 

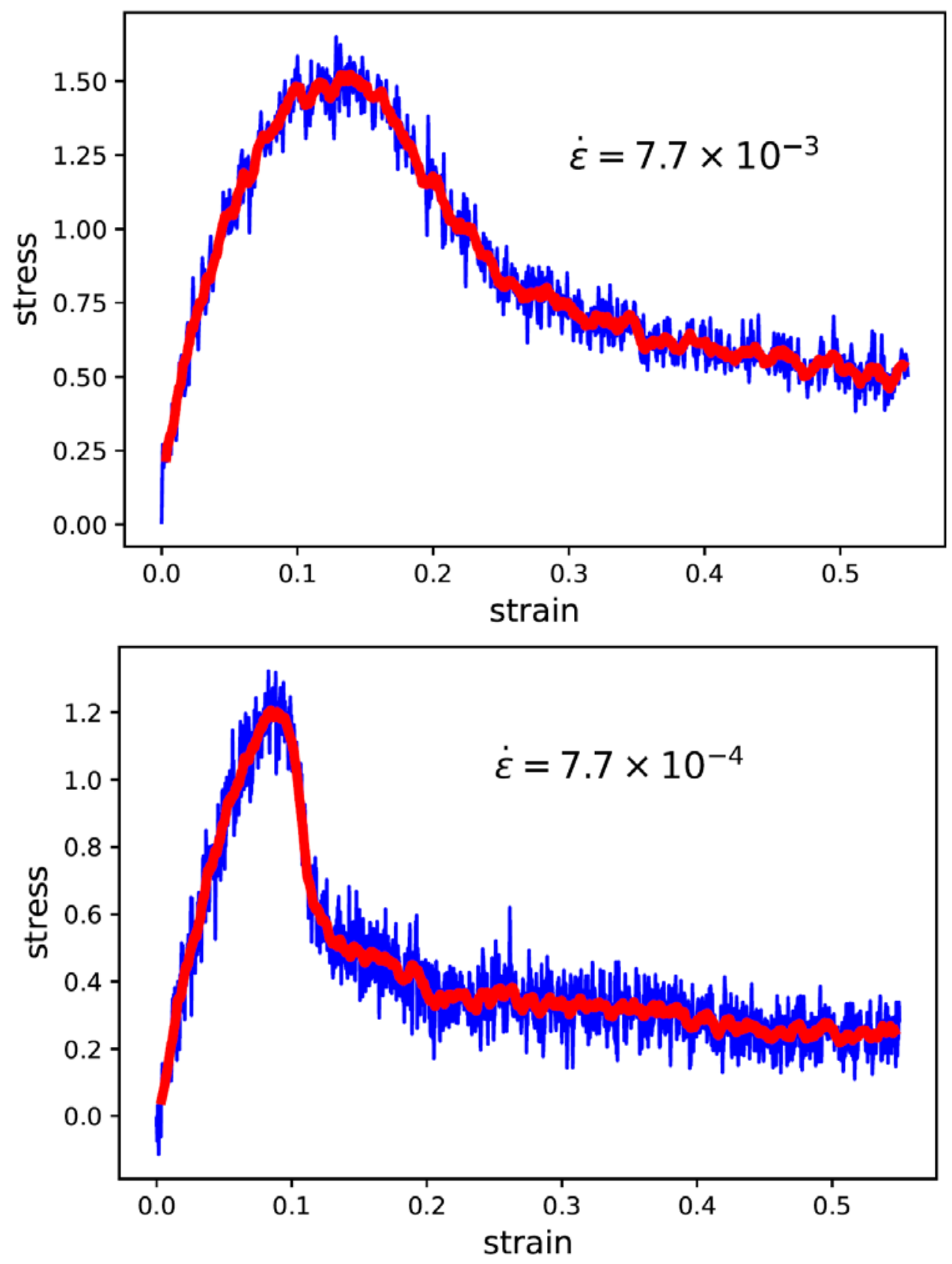

Figure SI.5: Initial stress-strain curves for the bulk polymer, for fast and slow deformations (above and below, respectively). The blue lines represent the instantaneous values, the red lines the batch or running averages over 100 timesteps (for $\dot{\varepsilon}=7.7 \times 10^{-3}$, corresponding to $\mathrm{v}=0.10$ ) or 1000 timesteps (for $\dot{\varepsilon}=7.7 \times 10^{-4}$, corresponding to $v=0.01$ ). The system contains 250 chains, with full threedimensional boundary conditions. Before carrying out the deformation, it was equilibrated at $T=1.0$ and $\mathrm{P}=0.0$. In analogy with the simulations of the confined polymer, the system is stretched the indicated rates along one direction (e.g., z), while leaving the periodicity along the orthogonal directions unchanged (i.e., imposing a Poisson ratio $\sigma=0.0$ ). 